\title{
Research on Parametric Regional Architecture Design Based on Ecological Perspective
}

\author{
Bo Zhang ${ }^{1, a}$ \\ ${ }^{1}$ LiaoNing Province Shenyang City University, Shenyang, Liaoning, China, 110112
}

Keywords: Ecology, Parametric, Regional Architecture

\begin{abstract}
Parametric design represents a new emerging design trend and opens up more room for today's design and development. As a result, a wide range of parametric buildings began to board the stage of history. In addition, in the design of the building, we also need to consider its specific context and environmental relations, in response to local geographical characteristics. At the same time, with the development of cultural diversity, national architects continue to actively explore the creation of the building with a regional temperament according to the terrain, sunshine and other natural element. Thus, the active development of regional culture, not only on the surface style, but also explore the cultural connotation of the building and it has become the core of the regional architecture. However, due to the current parametric design is still in the exploratory stage, parametric design reflects the blindness of the multi-cultural exploration at a certain extent. Therefore, in the ecological perspective, and based on the cultural diversity level, the parametric method for the exploration of regional architecture can establish a new paradigm of regional architecture and provide new opportunities for the establishment of new architectural aesthetics.
\end{abstract}

\section{Introduction}

By the development and influence of today's science and technology and philosophy, we gradually derived the parametric design with computer digital technology as the main carrier, and the parametric design method of Maya's MEL language and glasshopper became the mainstream of today's technology. The main use of parametric platform, by looking for the surrounding environment factors, to explore the logic between them and the relationship between the parameters as the starting point, the establishment of parametric model has become the main way of parametric design, and thus for the environment and building intervention to produce a New thinking. At the same time, with the development of globalization, contemporary architecture presents the opposite trend of designism with modernist architecture - no longer an internationalist style to embody the universality of architecture, and through cultural factors to highlight the cultural personality. Eventually it led to this perspective, the parametric eco-regional architecture and the formation of a new design paradigm.

\section{The Regional Trend of Parametric Buildings}

"Parametric design, that is, to find a specific relationship or rules, the impact of the design of the main factors organized together, where the factors that affect the design as a parameter or parameter, the formation of the parameter called the parameter model, and computer language Description, through the computer technology to parameter and variable data information into images, the result is the design of the embryonic". This information model, which is formed by the environmental parameters of the environment will result in the "structural modality tends to be the greatest degree of adaptation and coordination to the surrounding environment and human requirements" but also at the data level geographical environment factors to achieve the maximum fit. With the gradual implementation of parametric design and maturity, and gradually show the architect in the field of parametric areas of a large number of exploration of regional trends. Such as Chengdu Lanxi Ting to brick as a parameter of the water wall to the poetry of the parameters of the OCT headquarters office entrance design, extract the characteristics of the Qatar Petroleum Comprehensive District and other cases, are reflected in varying degrees, the use of parametric designer design Response to 
geographical features.

\section{The Ecological Regional Thinking Conversion of the Parametric Architecture}

Due to the parametric construction of the stage characteristics, as well as the parametric "by the emphasis on the results of the design into the process of attention to the design process" features, easily lead to the current architects on the parametric architectural form of excessive attention and resulting in formalism flooding tendency. But the evaluation of the building should not only be for the space is consistent with the focus on "space relations and color, light and other artistic atmosphere", but for a variety of factors on the complex system of consideration. Also in the field of parametric design, should also be a logical way to reflect the more rational for the environmental elements of care. Therefore, in the future parametric design, parametric to the environment and other elements of the logical conversion, resulting in ecological significance of the regional architecture, it should be a parametric development of the inevitable.

The Response of Natural Elements. Architecture cannot be separated from environmental factors and developed alone and the natural characteristics will be reflected in the architect's attitude to the geographical environment. But with the traditional design is different from the architect in the program design and energy consumption, the environment stage, prone to the application of data because of the ambiguity, and the relationship between building and environment, poor adaptability characteristics. Therefore, the design of the complex system to consider the design of the parameters, that is, through the airflow, lighting, sound field, sunshine and other simulation, the complex environmental factors to organize, the establishment of parameter model, so as to select the appropriate building layout, Generate changes with the epidermis, which provides a useful exploration for the relationship between architecture and environmental factors.
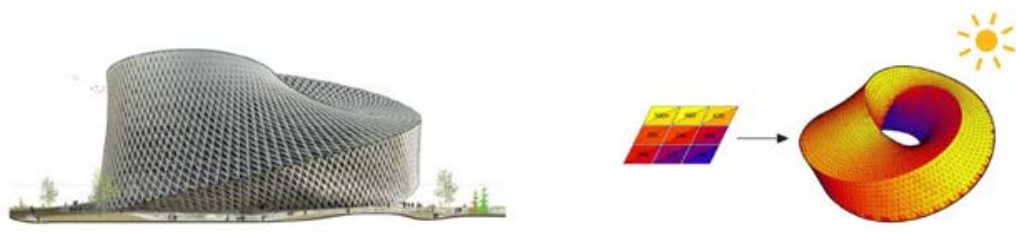

1 BIG office Astana National Library with architectural analysis of the skin

In the design of the Astana National Library (Figure 1), the architects, according to the regional nature of the environment, with Geco's environmental simulation platform, Nanjing University School of Architecture and Urban Planning Guo Fang in the Astana National Library the design of the window design in the energy-saving design of the relevant research introduced the building "with its daily average incident solar direct radiation (hereinafter referred to as thermal radiation) as a parameter to control the surface of the hole area, making the building room to obtain a more uniform of the amount of heat radiation, so as to achieve energy saving purposes ". According to the program design and building energy consumption simulation, improve the energy saving effect. Change the traditional design of the relationship between the building and the environment poor adaptability, in response to the natural environment of the parametric design to explore the road.

The Materials and Construction Methods. Human beings in the long-term survival and development, and constantly explore and life behavior, environmental characteristics of the material and space to adapt to the way, and ultimately produce a geographical environment characteristics of the building. Such as Xinjiang Ali Wang, Shanxi cave, etc., are all human repeated trial and error after the "optimization" results. This not only reflects the traditional spatial materials and means of building and regional context, cultural environment response, but also a positive ecological significance. And it is based on the foundation of the experience-based building.

In the field of parametric design, designers can also borrow materials and the way to create materials, and to create a means of building in the ecological role played by the parametric 
conversion attempt. As traditional buildings often use wood as the main material, in the parametric design of the exploration, the architects often use the wood "green" features, the design of the creation. Such as the Spanish Serbian parasol project, designed to show the form of honeycomb, through the fractal show the hierarchical structure of the building, reflecting the idea of the same body with the natural; overall shape shows a star-like structure, and the classic Spanish architecture harmony, echoed Serbia Regional characteristics of the area. In the selection of materials, to building materials, energy consumption, water pollution, air pollution and other environmental impact of the smallest environmentally friendly wood as the main timber, and in the wood outside the spraying of polyurethane coatings to meet the most stringent fire protection requirements. This kind of parameterized design means to material as a factor, by translation and to build the overall spatial form, has also become a new design of the new attempt.

The Evolution of the Form. The buildings are presented in solid form and are often closely linked to the geographical environment. Therefore, "creating a place of historical reference coordinates can bring a variety of ways for the development of design concepts" with appropriate body language, increase the user's sense of identity and sense of belonging. However, the relationship between the information and the description of the rules, often accompanied by a fuzzy and inaccurate, while the space comfort, environmental and other factors cannot give a good performance. Parametric design as a complex system of treatment, the key elements of the design elements of information conversion, simulation and production of buildings will reflect a designer under the control of the inevitable behavior. And the form also reflects the fit for the environment.

From the changing natural system to draw inspiration, to explore the environment to adapt to the form of generation, but also commonly used in the construction of the morphological logic for the building to the Aqua Tower, for example, through the calculation of parameters, Sun visor based on the line of sight, shade and other functions of the calculation, the formation of a natural feature of the facade system, so that the building embodies the Great Lakes region unique landscape texture of the characteristics of the limestone. At the same time designers to wave-like facade and select the convenience of birds to identify the gray mark with the sintered glass material, effectively prevent the impact of birds in North America, the problem of death, reflecting the ecological design of biological symbiosis (Figure 2,3 ).

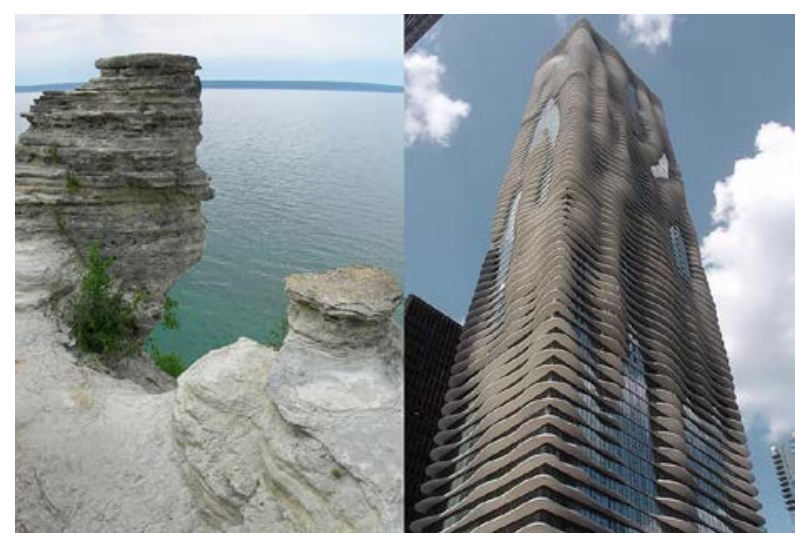

2 American Chicago limestone with Aqua Tower

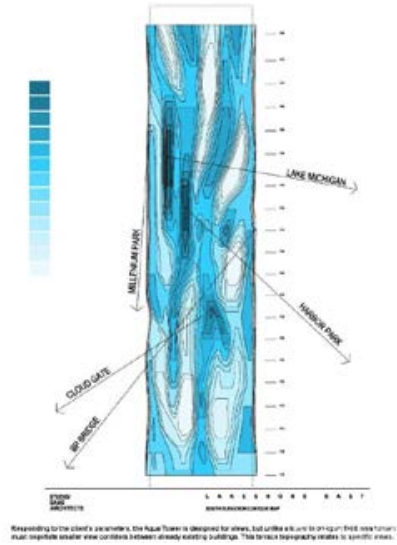

3 Aqua Tower skin parameters analysis

\section{The Ecological Exploration of Parametric Regional Architecture}

Under the influence of the complexity of science, the building reflects the complex and irregular characteristics of the space, and also determines the complex characteristics of the space form. At the same time, by the computer generated methodology for the building produced cross-era changes, but also greatly expands the designer's space thinking. The parametric design also utilizes its own advantages to embody the parametric design strategy of the architect, which will bring the advantages to the extreme and make meaningful and feasible exploration of the regionalized 
architecture in the ecological context. Learn from the attempt.

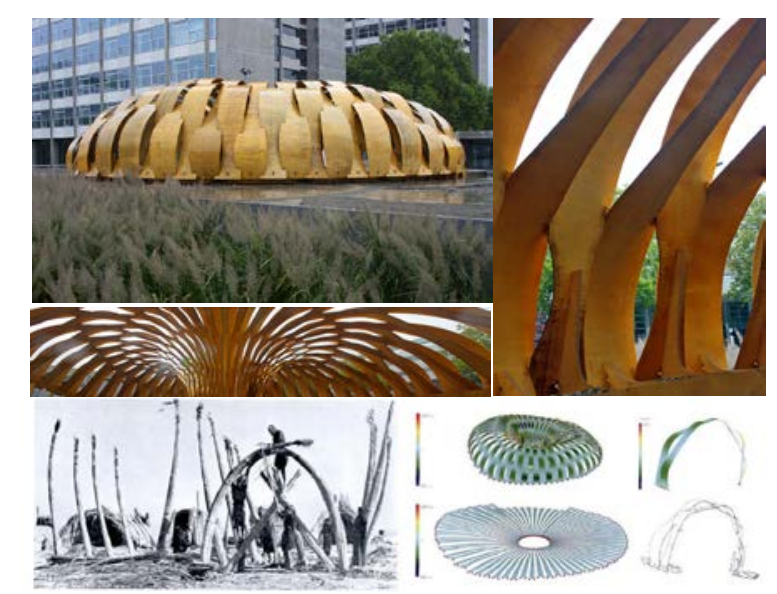

4 ICD / ITKE kiosk facade 5 ICD / ITKE kiosk internal structure 6ICD / ITKE kiosk detail 7MUDHIF and simulated deformation of the band-shaped components

The construction of the ICD / ITKE kiosks (Figure 4, 5, 6) at the University of Stuttgart in 2010 was based on a deep study of the construction of the reed method (Fig. 7) in the southern Iraqi man, Organizational style, and structural performance to define design parameters to generate space. Its physical and structural analysis is based on the characteristics of the natural environment, and sustainable wood eventually becomes the material choice for the ICD / ITKE kiosk. The success of the parametric design reflects the prospects of regional architectural design, to achieve the metaphysical ideology and metaphysical form of unity of the entity.

Another example is the Abu Dhabi Louvre Museum, where designers try to find a new design balance in tradition and modernity. By drawing the Arabian dome to enlarge, the formation of a $180 \mathrm{M}$ double dome building, and in the dome covered with free braided material, through the parametric model established to form a metaphor for the traditional pattern of Arab architecture (Figure 8, 9). Illuminated under the dome, forming a unique feeling of geographical characteristics, thus in the "national culture to extract elements to achieve parametric changes" (Figure 10) at the same time architects in the metaphor of geographical environment, trying to fit the context and the natural environment on the basis of, but also actively play a passive energy-saving potential.

Stone, steel and glass show three types of space. The three substances divide the different paths of light into the path and create a different translational procedural gradient. The shape of the building and the layout of the space play an important role for the formation of the construction of the wind field. Hollow dome to hollow woven material, taking into account the wind permeability, wind speed attenuation effect of small to avoid the wind valley, change the adverse wind field
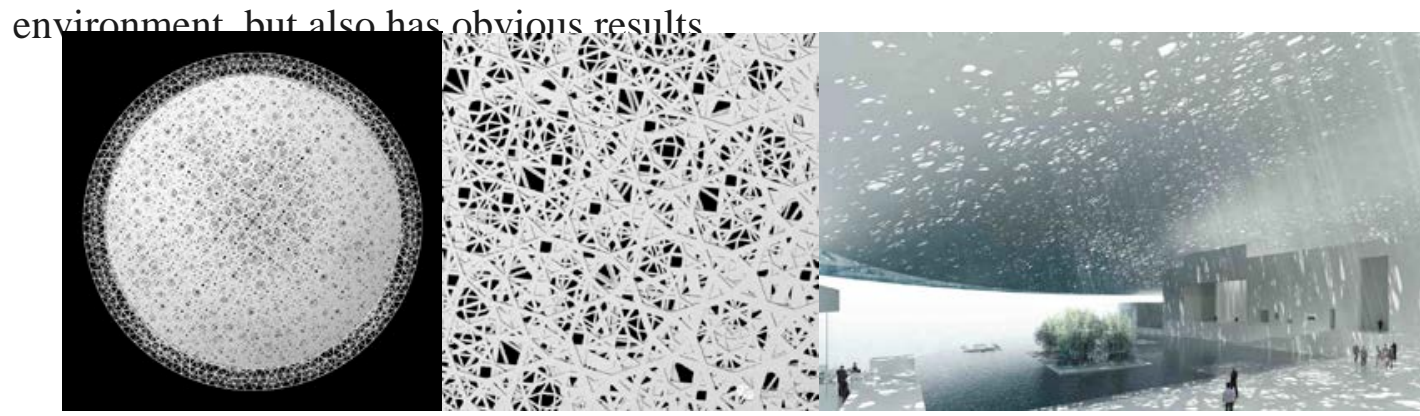

8 Abu Dhabi Louvre Museum Dome Plane 9 Braided Parametric Building Dome Detail 10 Abu Dhabi Louvre Museum Internal Perspective Effect

\section{The Parametric Regional Architectural Creation Road in the Ecological Context}

Diversified society as well as the computer as a means of open design, requiring the current designers to actively explore a fit of people's behavior, psychological habits and has a great 
environmental adaptability of the building, which has become the inevitable trend of the design. Therefore, in the investigation of the environment at the same time, still need to correctly understand the relationship between people - architecture - the environment, which not only need to pay attention to human behavior, but also in the design of the psychological level of meaning, and ultimately make the building in line with the local crowd living habits, geographical characteristics and geographical aesthetic characteristics.

From the One-Sided Form to the Overall Development. The current architectural form of flooding, the reason is that the parametric theoretical system has not yet established. In addition, because of our architects to some extent for the Western architectural theory of reference, but also led to the lack of theoretical basis and fall into the formalist misunderstanding. So the architectural design should go out only to stay in the history of the epidermis and experience analysis for the building. From the perspective of the whole life cycle of the building, to explore the parameters of the ecological aspects of the building for the thinking, correct the architect's values, improve the sense of responsibility of the architect and the design of consciousness.

Concerned about the Environmental Effects of Parametric Regional Buildings. Building is a large energy consumption, where the improper handling of the building, will have an adverse impact on the environment. Blind construction and demolition, the result will only cause for the architectural form of play, resulting in the neglect of the environmental benefits of space. At the same time, the relationship between architecture and the environment will be seriously blocked, but also cause a range of degradation of ecosystem functions. Therefore, for the current parametric regional construction, should take the ecological pattern of evolution and construction of the construction and expansion of the combination of parametric geographical architecture placed in the environmental system to consider the overall relationship between the organic whole, so as to improve the living environment and environmental environment quality, and comprehensively improve the ecological service value of parametric regional buildings.

Improve the Sustainable Environmental Assessment System. The concept of "ecology, green" was raised in the 1970s, but the theory of practice is still in its infancy. Due to the lack of a sound sustainable environmental assessment system and even lead to some extent there is "green design" errors. Improve the sustainable environmental assessment system, not only taking into account the regional impact on the green building results, but also the need for scientific means, the sustainable development of the building as a foothold. This requires not only the community, including the user, the designer and the construction of multi-party cooperation and common participation, but also the need for designers to eco-design methods as a technical support to establish a clear data analysis, evaluation indicators and strict quality certification to ensure the healthy and orderly development of the construction market has important guiding significance.

\section{Conclusion}

Digital media technology in the information age provides a new perspective and useful architectural thinking exploration for the future development of regional architecture in the process of regional culture and heritage. However, in the response to global cultural convergence phenomenon and the possibility of regional culture to explore the possibility of whether the geographical construction should also be placed in the ecological balance of perspective to explore and explore the possibility of more green areas of the building? The development of science and technology is not the form of a formal approach to form, the parametric design is not the proliferation of formalism, "culprit." Fragmented environment, consider the form of regional construction in the mechanical point of view, in the sense of environmental significance, it will only show its vulnerability. The world is complex and dynamic. Therefore, as a regional architecture, explore the ecological perspective of the complex science-based parametric design is the reasonable way out of regional construction and rational return in the future. Using parametric means to explore the ecological design of regional architecture is also a new attempt for the regional culture. 


\section{References}

[1] Xu Weiguo, Xu Feng "Urban Architecture" editorial department. Parametric design in Chinese architectural creation and thinking - Tsinghua University architecture College Professor Xu Weiguo, Mr. Xu Feng interview. Urban architecture, 2010.06: 108-112

[2] Xu Weiguo. Dialogue Xu Weiguo. Urban Environment Design, 2010.10: 15

[3] Xu Weiguo, Huang Weixin, Jin Mingyu. Process logic - the technical route of "nonlinear architecture design".Built .2010.06: 10-14

[4] Zhou Haoming. Sustainable indoor environment design theory. Beijing: China Construction Industry Press .2011.01: 53

[5] Zeng Peng, Cai Liangwa. Parametric regional architectural creation method research. New construction .2014.06: (112-116)

[6] [Germany] Belt Bielefield, [West] Bastion El Curi. Design concept. Zhang Lufeng translation. Beijing: China Construction Industry Press 2010.01: 106

[7] Yang Zifu, Sun Cheng. Regionalized expression in parametric architectural design. Simulation • Coding - Collaboration - 2012 National Institute of Architecture. Department of Architecture Digital Technology Teaching Seminar .2012.08: 62-67

[8] [Taiwan] Lin Xiande. Green building. Beijing: China Construction Industry Press .2011.11: 78-105

[9] Zhang Fan, Xing Kai, Liang Jing. Research on the parametric design of green building based on environmental parameters. Digital construction culture - 2015 National Architecture Department of Digital Technology Teaching Seminar, 2015.06: 116-119

[10] Xu Weiguo. Parametric design and algorithm formation. World Architecture .2011.06: $110-111$

[11] Wu Zhiqiang .2005 / 2006 the first Holcim Sustainable Building Grand Prix winning entries. Beijing: China Construction Industry Press .2007.04: (86-89)

[12] Li Yide. Digital technology in the context of the combination of local architectural design construction. Dalian University of Technology master's degree thesis .2013.06

[13] Xu Weiguo. Digital construction. Journal of Architecture .2009.01: 61-63

[14] Guo Fang. Geco in the parametric building energy-saving design application - Kazakhstan Astana National Library window hole design as an example. Urban construction .2013.03: 222

[15] Liu Bo, Guo Jinsheng, Yan Zhibin.Study on the Exploitation of the Ecological Energy Conservation Potential of the Decorative Parametric Buildings. Digital Engineering DADA2015 Series International Symposium on Digital Architecture Digital Engineering .2015.07: 223-231

[16] Mei Hongyuan, Zhang Xiangning, Zhu Ying. Return to the origin of contemporary Chinese regional architectural creation. Journal of Architecture .2010.11: 106-109

[17] Liu Xianjue. Ecological Architecture. Beijing: China Construction Industry Press .2009.05: 501-587

[18] Patrick Schumacher, Neil Ritchie, Guo Lei. On the parametric Neil Ritchie vs. Patrick Schumacher. Time Building .2012.09: 32-35

[19] Shi Chaogang.Study on Green Building Evaluation System Based on Sustainable Development. Master's Thesis of Hunan University .2007.10: 
[20] Chen Liding, Sun Ranhao, Liu Hailian.Study on the ecological environment effect of urban landscape pattern evolution. Acta Ecologica Sinica 2013.02: 1042-1050 